\title{
Early perturbation in mitochondria redox homeostasis in response to environmental stress predicts cell fate in diatoms
}

\author{
Shiri Graff van Creveld ${ }^{1,3}$, Shilo Rosenwasser ${ }^{1,3}$, Daniella Schatz ${ }^{1}$, Ilan Koren ${ }^{2}$ and Assaf Vardi ${ }^{1}$ \\ ${ }^{1}$ Department of Plant Sciences, Weizmann Institute of Science, Rehovot, Israel and ${ }^{2}$ Department of Earth and \\ Planetary Sciences, Weizmann Institute of Science, Rehovot, Israel
}

\begin{abstract}
Diatoms are ubiquitous marine photosynthetic eukaryotes that are responsible for about $20 \%$ of global photosynthesis. Nevertheless, little is known about the redox-based mechanisms that mediate diatom sensing and acclimation to environmental stress. Here we used a redox-sensitive green fluorescent protein sensor targeted to various subcellular organelles in the marine diatom Phaeodactylum tricornutum, to map the spatial and temporal oxidation patterns in response to environmental stresses. Specific organelle oxidation patterns were found in response to various stress conditions such as oxidative stress, nutrient limitation and exposure to diatom-derived infochemicals. We found a strong correlation between the mitochondrial glutathione (GSH) redox potential $\left(E_{G S H}\right)$ and subsequent induction of cell death in response to the diatom-derived unsaturated aldehyde $2 \mathrm{E}, 4 \mathrm{E} / \mathrm{Z}$-decadienal (DD), and a volatile halocarbon (BrCN) that mediate trophic-level interactions in marine diatoms. Induction of cell death in response to DD was mediated by oxidation of mitochondrial $\mathrm{E}_{\mathrm{GSH}}$ and was reversible by application of GSH only within a narrow time frame. We found that cell fate can be accurately predicted by a distinct life-death threshold of mitochondrial $\mathrm{E}_{\mathrm{GSH}}(-335 \mathrm{mV})$. We propose that compartmentalized redox-based signaling can integrate the input of diverse environmental cues and will determine cell fate decisions as part of algal acclimation to stress conditions.
\end{abstract}

The ISME Journal (2015) 9, 385-395; doi:10.1038/ismej.2014.136; published online 1 August 2014

\section{Introduction}

Production of reactive oxygen species (ROS) in photosynthetic organisms occurs mainly in the chloroplast and mitochondria as a byproduct of oxygen-based metabolism (Halliwell, 2006). As ROS can be highly toxic molecules, their levels are tightly regulated by the antioxidant system that consists of ROS-scavenging enzymes and small molecules, such as glutathione (GSH) (Mittler, 2002; Foyer and Noctor, 2011). Although toxic ROS levels are controlled by the cellular antioxidant system to prevent oxidative damage, moderate ROS levels can be used as central secondary messengers that regulate signaling networks (Danon, 2002; D'Autréaux and Toledano, 2007; Jones and Go, 2010). The specificity of the ROS signal is achieved by variations in ROS levels, the specific chemical species and their intracellular localization (Foyer and Noctor, 2003; Gadjev et al., 2006; Møller and Sweetlove, 2010; Rosenwasser et al., 2013, 2014).

$\mathrm{GSH}$, the most abundant low-molecular-weight thiol antioxidant, has a critical role in maintaining a

Correspondence: A Vardi, Department of Plant Sciences, Weizmann Institute of Science, Rehovot, 76100, Israel.

E-mail: assaf.vardi@weizmann.ac.il

${ }^{3}$ These authors contributed equally to this work.

Received 12 January 2014; revised 12 June 2014; accepted 18 June 2014; published online 1 August 2014 reducing cellular thiol-disulfide balance and in detoxification of $\mathrm{H}_{2} \mathrm{O}_{2}$ via the ascorbate-GSH cycle (Rijstenbil and Wijnholds, 1996; Foyer and Noctor, 2011). In addition, GSH was recently suggested to have a pivotal role in regulating iron metabolism by maintaining the mitochondrial iron-sulfur clusters in yeast (Kumar et al., 2011). Oxidative stress that results from imbalance between ROS generation and antioxidant capacity is a common feature of cells exposed to environmental stress conditions. Under such conditions, electrons used for ROS detoxification are drawn, at least in part, from the cellular GSH pool, leading to an elevation of oxidized glutathione and a shift in the GSH redox potential $\left(\mathrm{E}_{\mathrm{GSH}}\right)$ toward oxidation (Meyer, 2008). Alterations in the $\mathrm{E}_{\mathrm{GSH}}$ as a result of ROS production can regulate the activation of many biological processes, such as transcription, post-translational modification and protein-protein interactions, by affecting the oxidation state of thiol groups in redoxsensitive proteins (Dietz, 2008; Meyer, 2008).

The development of fluorescent redox-sensitive biosensors such as the reduction-oxidation sensitive green fluorescent protein (roGFP) (Dooley et al., 2004; Hanson et al., 2004) allows in vivo quantitative and dynamic measurements of the cellular redox state and offers an advantage over other common destructive methods (for example, crude cell extracts). In vitro characterization of roGFP activity 
in plants, showed that roGFP reduction is mediated by glutaredoxin, which catalyzes the reversible electron flow between GSH and target proteins (Meyer et al., 2007). In contrast, the redox status of roGFP was not significantly affected by thioredoxin or other redox-active compounds such as nicotinamide adenine dinucleotide phosphate and ascorbate (Meyer et al., 2007; Gutscher et al., 2008). These in vitro observations were also tested in vivo and the specificity of the roGFP to detect the redox state of the GSH pool was validated in HeLa cells, using GSH biosynthesis inhibitors (Dooley et al., 2004) and in Arabidopsis mutants in GSH biosynthesis and reduction (Meyer et al., 2007; Rosenwasser et al., 2010). In addition, in vitro analysis showed that the fluorescence ratio data obtained from the roGFP is insensitive to alteration of the $\mathrm{pH}$ in physiological range (Schwarzländer et al., 2008). The above study points for the predominant interaction between the roGFP and the GSH pool and suggests that $\mathrm{E}_{\mathrm{GSH}}$ can be assessed in vivo in various cellular organelles by roGFP.

Diatoms are a highly predominant group of eukaryotic photosynthetic alga responsible for about $40 \%$ of marine photosynthesis (Nelson et al., 1995; Field et al., 1998). Consequently, diatoms have a central role in the biogeochemical cycling of important nutrients such as carbon and nitrogen. Diatoms can form massive blooms in the ocean that are controlled by abiotic stress, such as nutrient limitation and light regime, and by biotic interactions with grazers, viruses and through allelopathic interactions. During these interactions, an array of bioactive compounds (infochemicals) that have an important role in regulating cell fate are produced, and can shape population dynamics and composition (Pohnert et al., 2007). When diatom populations are subjected to grazing or nutrient stress, cells can rapidly induce the biosynthesis of diatom-derived oxylipins (DD) or volatile halocarbons (BrCN), which are bioactive signals that act as chemical defense mechanisms against grazing or as allelochemicals to suppress competing species (Ianora et al., 2004; Casotti et al., 2005; Vardi et al., 2006; Vanelslander et al., 2012). Recent reports suggest that diatom cells may utilize a $\mathrm{Ca}^{2+}$ induced NO-based system to monitor stressed cells within a population. In this system, the non-stressed cells monitor the level of DD concentrations produced by the stressed cells, thereby providing a stress surveillance system to monitor the stress level of the entire population (Vardi et al., 2006, 2008). Release of sublethal levels of DD can serve as an early warning protective mechanism, and lethal doses will initiate the cascade responsible for cell death and bloom termination. Recent evidences suggested that diatoms induce hallmarks of a programmed cell death (PCD)-like mechanism as a result of environmental stress such as iron limitation, culture age and exposure to infochemicals (Berges et al., 1998; Casotti et al., 2005; Vardi et al.,
2008; Thamatrakoln et al., 2012). Nevertheless, very little is known about the role of ROS in mediating the perception of environmental stress conditions and possible regulation of cell fate during algal bloom dynamics.

We hypothesized that various environmental stress conditions may differentially affect the subcellular $\mathrm{E}_{\mathrm{GSH}}$, which will consequently decode this redox perturbation into activation of specific signal transduction pathways that regulate cell fate. To examine this hypothesis, we monitored subcellular $\mathrm{E}_{\mathrm{GSH}}$ values in the model diatom Phaeodactylum tricornutum under steady-state and stress conditions by using diatom cells expressing the roGFP probe in various subcellular localizations. We provide compelling evidence for the specificity in diatom's response to oxidative stress and multiple environmental cues by alteration of organelle-specific $\mathrm{E}_{\mathrm{GSH}}$. Our results also show a strong correlation between early redox perturbation in the mitochondria and subsequent cell death, and reveal that cell death can be predicated based on a distinct mitochondrial $\mathrm{E}_{\mathrm{GSH}}$ threshold. These results may have important implications for better understanding the cellular mechanisms used by marine diatoms during acclimation to stress and the fate of blooms in the ocean.

\section{Materials and methods}

\section{Culture growth}

P. tricornutum, accession Pt1 8.6 (CCMP2561 in the Provasoli-Guillard National Center for Culture of Marine Phytoplankton) was purchased from the National Center of Marine Algae and Microbiota (NCMA, formerly known as CCMP; Bigelow, ME, USA). Unless otherwise specified, cultures were grown in $\mathrm{f} / 2$ media $\left(882.4 \mu \mathrm{M} \mathrm{NaNO}_{3}, 35.21 \mu \mathrm{M}\right.$ $\mathrm{Na}_{2} \mathrm{HPO}_{4} \bullet 12 \mathrm{H}_{2} \mathrm{O}, \quad 1.83 \mu \mathrm{M} \quad \mathrm{FeCl}_{3} \bullet 6 \mathrm{H}_{2} \mathrm{O}$ ) (Guillard and Ryther, 1962) at $18{ }^{\circ} \mathrm{C}$ with $16: 8 \mathrm{~h}$ light:dark cycles and light intensity of $80 \mu \mathrm{mol}$ photons $\mathrm{m}^{-2}$ $\mathrm{s}^{-1}$ supplied by cool-white LED lights (Edison, New Taipei, Taiwan). All experiments were performed with exponentially growing cultures at $\sim 5 \times 10^{5}$ cells ml ${ }^{-1}$. Nutrient limitation experiments were done by washing the cells in nutrientfree media and resuspending them in $\mathrm{f} / 2$ without the appropriate nutrient (nitrogen or iron). For iron limitation, $1 \mu \mathrm{M}$ of the efficient iron chelator desferrioxamine $\mathrm{B}$ was added.

\section{Fluorescence microscopy}

Microscopy images where obtained on IX71S1F-3-5 motorized inverted Olympus microscope (Tokyo, Japan) equipped with a $60 \times$ objective and filter systems for oxidized roGFP (ex:405/20 nm, em:525/ $50 \mathrm{~nm}$ ), reduced roGFP, Annexin V, TUNEL and sytox staining (ex:470/40 nm, em:525/50 nm), and chlorophyll autofluorescence (ex:500/20 nm, em:650 nm LP). Images were captured using an EXi Blue (Q Imaging, Surrey, BC, Canada) camera. 


\section{Measuring roGFP oxidation}

Using fluorescence microscopy, oxidized and reduced forms of roGFP were observed at excitation of $405 \mathrm{~nm}$ and $470 \mathrm{~nm}$, respectively. In all, $525 \mathrm{~nm}$ emission intensities were collected and ratio images were created by dividing the ex:405, em:525 nm image by the ex:470, em:525 nm image, pixel by pixel using MATLAB, displayed in pseudocolor. Quantitative data were obtained using an Eclipse iCyt flow cytometer (Sony Biotechnology Inc., Champaign, IL, USA), equipped with 405 and $488 \mathrm{~nm}$ solid state air cooled lasers, both with $25 \mathrm{~mW}$ on the flow cell and with standard filter set-up, whereby roGFP was measured in the green channel $(525 / 50 \mathrm{~nm})$ following excitation at $405 \mathrm{~nm}$ (oxidized) and $488 \mathrm{~nm}$ (reduced). Leakage of chlorophyll autoflorescence to the green channel was subtracted by measurement of WT cells in parallel to roGFP transformants. Calibration of the fluorescence ratio arbitrary units was achieved by comparing the ratios of the roGFP fully reduced and fully oxidized states $(30 \mathrm{~min}$ after treatment with $1 \mathrm{~mm}$ DTT or $200 \mu \mathrm{M} \mathrm{H}_{2} \mathrm{O}_{2}$, respectively). The degree of oxidation of roGFP and $\mathrm{E}_{\mathrm{GSH}}$ values were calculated according to Schwarzländer et al. (2008) using the Nernst equation and $\mathrm{pH}$ values of 6.9 for the cytoplasm and nucleus, 7.9 for the chloroplast (Anning et al., 1996) and 7.8 for mitochondria (Schwarzländer et al., 2008). Alterations of the estimated $\mathrm{pH}$ values will alter the calculated redox potential by $2.9 \mathrm{mV}$ for every $0.05 \mathrm{pH}$ units (Supplementary Figure S1). Kinetic analyses of degree of oxidation of roGFP were performed, cells were kept under light intensity of $20 \mu \mathrm{mol}$ photons $\mathrm{m}^{-2} \mathrm{~s}^{-1}$ throughout the measurements.

GSH reduced ethyl ester (Sigma-Aldrich, Rehovot, Israel) was added to the cells at a final concentration of $1 \mathrm{~mm}$.

\section{Results and Discussion}

Compartmentalized basal redox state in P. tricornutum In order to map the in vivo spatial and temporal redox patterns in the model diatom $P$. tricornutum under steady state and in response to environmental stresses, the redox-sensitive GFP sensor was targeted to specific organelles, the cytoplasm (cyt-roGFP), the chloroplast stroma (chl-roGFP), the nucleus (nuc-roGFP) and the mitochondria (mit-roGFP) (Rosenwasser et al., 2014).

Ratiometric images derived from the division of the emitted fluorescence (measured at $525 \mathrm{~nm}$ ) due to excitation at $405 \mathrm{~nm}$ (Figure 1a) and $488 \mathrm{~nm}$ (Figure 1b) are indicative of the in vivo oxidation

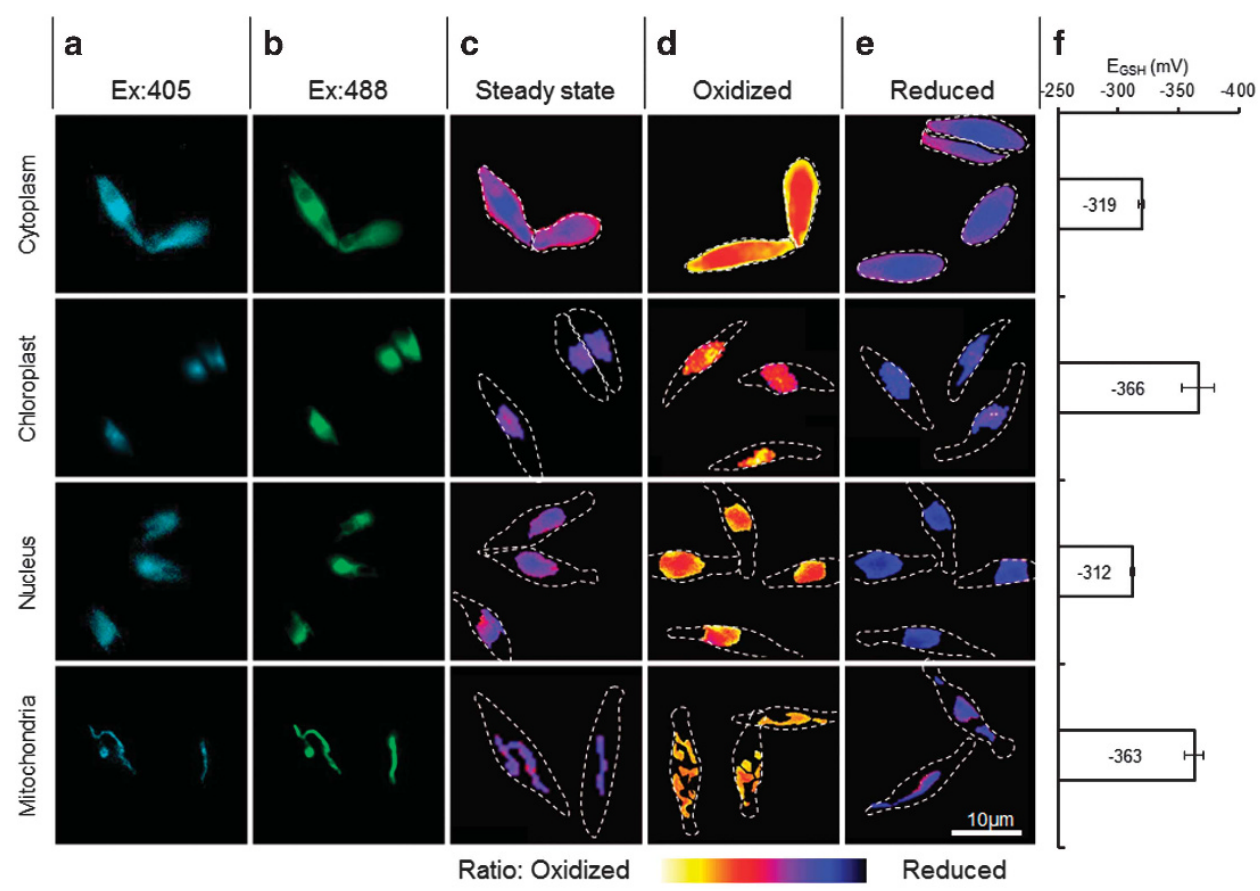

Figure 1 In vivo imaging of subcellular redox potential in P. tricornutum cells. Fluorescence microscopy imaging of $P$. tricornutum cells expressing roGFP2 in various subcellular localizations. Each row represent images depicted from each organelle; cytoplasm, chloroplast, nucleus and mitochondria. Fluorescence images of oxidized roGFP (ex:405 nm, em:525 nm) (a), and reduced roGFP (ex:488 nm, em:525 nm) (b), were divided to obtain the ratiometric images under steady-state condition (c). Ratiometric images of cells exposed to an oxidant ( $\left.150 \mu \mathrm{M} \mathrm{H}_{2} \mathrm{O}_{2}\right)$ (d) and reductant (1 mM DTT) (e) are presented. Dashed lines represent the cells' outline, drawn based on the bright field images. Scale bar: $10 \mu \mathrm{m}$. (f) Steady-state $\mathrm{E}_{\mathrm{GSH}}$, in $\mathrm{mV}$, was calculated based on the Nernst equation using the oxidation level under given $\mathrm{pH}$ values for each organelle. $\mathrm{E}_{\mathrm{GSH}}$ values were calculated based on averaged flow cytometry fluorescence measurements of at least 5000 cells per sample and presented as mean \pm s.d., $n=3$. Representative data from at least three independent experiments, each containing three biological replicates, are shown. 
level of roGFP (Figures 1c-e; Dooley et al., 2004; Hanson et al., 2004). Fluorescence ratios (ex:405, em:525 nm/ex:488, em:525 nm) obtained following oxidation by $\mathrm{H}_{2} \mathrm{O}_{2}$ were higher than in steady-state cells in all the examined subcellular compartments (Figures 1d and c). On the other hand, the fluorescence ratios of the images obtained after application of an external reductant (DTT) were only slightly lower than the steady-state ratios, indicating that, under steady-state conditions, all the examined compartments (cytoplasm, nucleus, mitochondria and chloroplast) have highly reduced microenvironments (Figures 1e and c). The dynamic range of the probe $\left(\right.$ Ratio $_{\text {oxidized }} /$ Ratio $\left._{\text {reduced }}\right)$, calculated based on flow cytometry measurements, was 6.8-9.3, similar to the that obtained from purified roGFP2 (Schwarzländer et al., 2008). These data demonstrate high in vivo signal-to-noise ratios and suggest that the roGFP probe can serve as an accurate sensor for subcellular $E_{G S H}$ in marine diatoms.

Based on the roGFP ratio data, the $\mathrm{E}_{\mathrm{GSH}}$ values in the different subcellular compartments can be estimated using the Nernst equation (Meyer and Dick, 2010). We assumed $\mathrm{pH}$ values of 6.9 for the cytoplasm and nucleus, 7.9 for the chloroplast based on measurements in coccolithophorids (Anning et al., 1996) and 7.8 for mitochondria based on measurements in plants (Schwarzländer et al., 2008). Importantly, despite the $\mathrm{pH}$-independent characteristics of the roGFP sensor, alterations of the estimated $\mathrm{pH}$ values will alter the calculated redox potential by $2.9 \mathrm{mV}$ for every $0.05 \mathrm{pH}$ units (Supplementary Figure S1) as derived from the Nernst equation. The $\mathrm{E}_{\mathrm{GSH}}$ in the cytoplasm and nucleus were similar and ranged between -319 and $-312 \mathrm{mV}$, while that of the mitochondria and chloroplast were more reduced, reaching -363 and $-366 \mathrm{mV}$ (Figure 1f). These results show that the GSH pool is kept under distinct, non-equilibrium reduced state in each subcellular compartment. Similar results describing the compartmentalization of the $\mathrm{E}_{\mathrm{GSH}}$ steady-state conditions were reported for mammalian cells and plants (Hanson et al., 2004; Hu et al., 2008; Schwarzländer et al., 2008; Rosenwasser et al., 2010; Dardalhon et al., 2012).

\section{Organelle-specific response to oxidative stress in diatom cells}

We further examined the differential response of the $\mathrm{E}_{\mathrm{GSH}}$ in the various organelles by kinetic measurements of oxidation and reduction of roGFP following $\mathrm{H}_{2} \mathrm{O}_{2}$ treatments (Figure 2a). All transformants harboring the roGFP probe oxidized in a dosedependent manner and responded to physiologically relevant concentrations of $\mathrm{H}_{2} \mathrm{O}_{2}$ (Figure 2). However, saturation of the probe in each of the organelles was reached at different $\mathrm{H}_{2} \mathrm{O}_{2}$ concentrations. The cyt-roGFP reached saturation at concentrations of $50-80 \mu \mathrm{M} \mathrm{H}_{2} \mathrm{O}_{2}$, nuc-roGFP reached saturation at
80-100 $\mu \mathrm{M} \mathrm{H}_{2} \mathrm{O}_{2}$, while the mitochondria and chloroplast probes saturated at $150 \mu \mathrm{M} \mathrm{H}_{2} \mathrm{O}_{2}$ (Figures 2b-e). Interestingly, we detected only minor oxidation $(\sim 5 \%)$ between 0 and $50 \mu \mathrm{M} \mathrm{H}_{2} \mathrm{O}_{2}$ in the chloroplast, while a high oxidation level $(>50 \%)$ was detected for $50 \mu \mathrm{M} \mathrm{H}_{2} \mathrm{O}_{2}$ treatment in all other roGFP transformants (Figures 2b-e). These data may indicate an organelle-specific antioxidant capacity to cope with oxidative stress and ROS-mediated stress conditions. We further utilized the reversibility property of the roGFP probe to monitor the in vivo oxidation-reduction patterns over time of exposure to oxidative stress. Once oxidized, the roGFP probe may be reduced back to its basal level. We termed this dynamic the recovery phase. Differential patterns of the recovery phase were observed in each of the examined subcellular compartments in response to a range of $\mathrm{H}_{2} \mathrm{O}_{2}$ concentrations. Although roGFP oxidation in the cytoplasm and nucleus recovered from exposure to 50-80 $\mu \mathrm{M} \mathrm{H}_{2} \mathrm{O}_{2}$ (Figures $2 \mathrm{~b}$ and d), the oxidation in the chloroplast only partially recovered and did not reduce back to the basal state (Figure 2c). A unique pattern of recovery was observed in the mitochondrial roGFP, which fully recovered by $4 \mathrm{~h}$ after the addition of up to $150 \mu \mathrm{M} \mathrm{H}_{2} \mathrm{O}_{2}$ (Figure 2e). Importantly, addition of $1 \mathrm{~mm}$ reduced GSH $30 \mathrm{~min}$ after the treatment with $150 \mu \mathrm{M} \quad \mathrm{H}_{2} \mathrm{O}_{2}$ quickly reduced the probe back to its basal level in all organelles (Supplementary Figure S2), emphasizing the sensitivity and reversibility of the roGFP probe in its ability to detect perturbations in cellular GSH pool. Taken together, these data demonstrate the compartmentalized characteristics of the $\mathrm{E}_{\mathrm{GSH}}$ in different organelles within a diatom cell.

Early redox perturbation in response to oxidative stress can predict cell fate

Widespread documentation of PCD in diverse classes of phytoplankton, including chlorophytes, dinoflagellates, diatoms and coccolithophorids, in response to environmental stresses suggest an important evolutionary and ecological role for PCD in marine protists (Bidle and Falkowski, 2004; Franklin et al., 2006; Orellana et al., 2013). Nevertheless, the molecular mechanisms involved in PCD in phytoplankton are poorly understood. We used the roGFP transformants to examine a possible role of $\mathrm{E}_{\mathrm{GSH}}$ oxidation in mediating PCD in diatoms. Application of $\mathrm{H}_{2} \mathrm{O}_{2}$ induced cell death that was characterized by DNA degradation (detected by the TUNEL assay), externalization of phosphatidylserine (Annexin V staining) and compromised cell membrane (Sytox Green staining) (Figures 3a-i). Application of reduced GSH to cells treated with a lethal $\mathrm{H}_{2} \mathrm{O}_{2}$ dose $(150 \mu \mathrm{M})$ rescued the cells from induction of cell death and led to full recovery of the $E_{G S H}$ in all examined organelles (Figure 3j, Supplementary Figure S2). Intriguingly, we identified a narrow time frame in which GSH addition rescued 
a

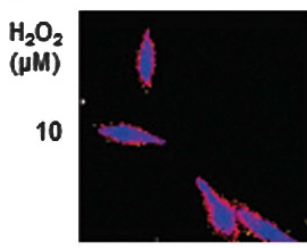

150

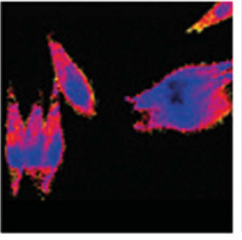

Steady state
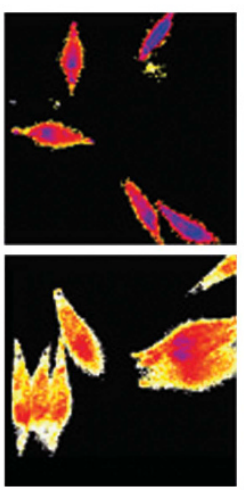

1
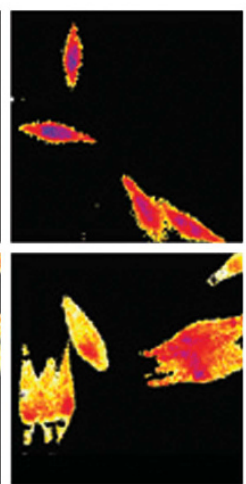

16
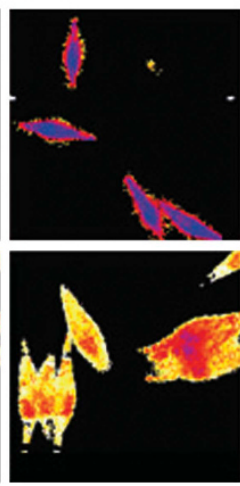

32

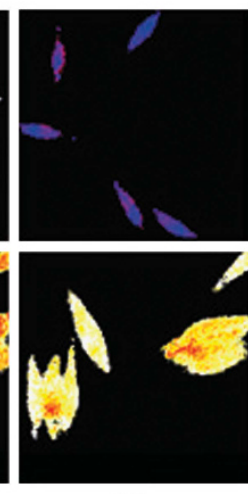

64

Time after addition of $\mathrm{H}_{2} \mathrm{O}_{2}$ (min)

b

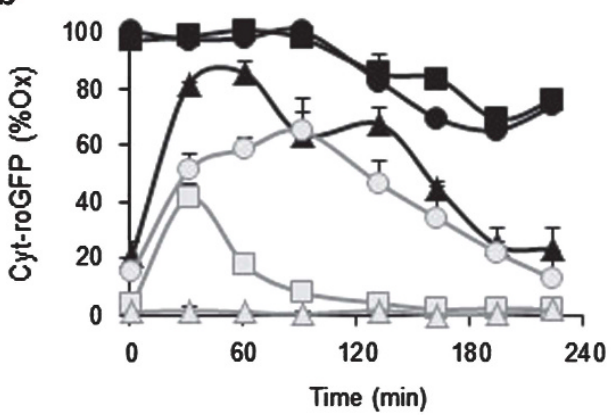

d

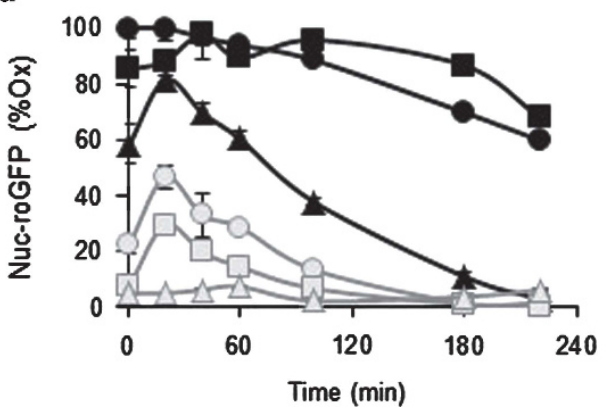

C

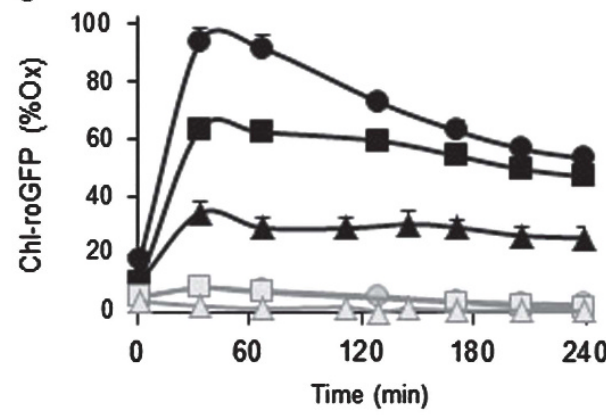

e

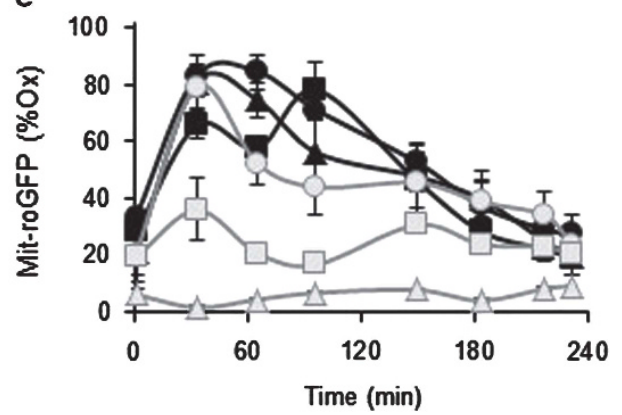

Oxidized

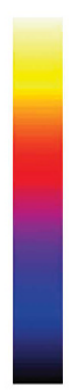

Reduced
$\mathrm{H}_{2} \mathrm{O}_{2}$

( $\mu \mathrm{M})$

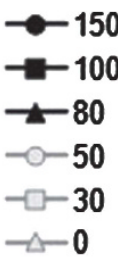

Figure 2 Organelle-specific oxidation patterns in response to oxidative stress. (a) Ratiometric imaging of cells expressing cyt-roGFP under steady-state conditions and after exposure to 10 or $150 \mu \mathrm{M} \mathrm{H}_{2} \mathrm{O}_{2}$. (b-e) Dose-dependent degree of oxidation of roGFP as detected during $4 \mathrm{~h}$ after application of a range of $\mathrm{H}_{2} \mathrm{O}_{2}$ concentrations: 0, 30, 50, 80, 100 and $150 \mu \mathrm{M}$ (gray triangles, squares, circles, black triangles, squares, circles respectively). roGFP degree of oxidation in the cytoplasm (b), chloroplast (c), nucleus (d) and mitochondria (e) are presented. roGFP degree of oxidation values were calculated based on averaged flow cytometry fluorescence measurements of at least 5000 cells per sample and presented as mean \pm s.d., $n=3$. Representative data from at least three independent experiments, each containing three biological replicates, are shown.

the cells from the lethal dose of $\mathrm{H}_{2} \mathrm{O}_{2}$. Addition of GSH $2 \mathrm{~h}$ or more after oxidation resulted in cell death $24 \mathrm{~h}$ after treatment (Figure 3j), despite the reduction of the organelle microenvironment. These results suggest an early critical phase in the subcellular response to redox perturbation induced by $\mathrm{H}_{2} \mathrm{O}_{2}$ and its subsequent regulation of cell fate. The inability of GSH to rescue cells after $2 \mathrm{~h}$ of oxidative stress could point at the induction of an irreversible signal transduction cascade that regulates cell death following perturbations in the $\mathrm{E}_{\mathrm{GSH}}$.
Mitochondrial redox perturbation can predict cell fate under diverse environmental stress conditions

During algal bloom succession, diatom cells are subjected to diverse stress conditions at different phases of the bloom, from initiation to demise. As numerous diverse environmental stress conditions lead to increased ROS production, a key question is how specificity in signaling allows deciphering between multiple stressors and specific phenotypic responses (Mittler et al., 2004; Gadjev et al., 2006; Rosenwasser et al., 2013). To examine if environmental stresses differentially affect the subcellular 

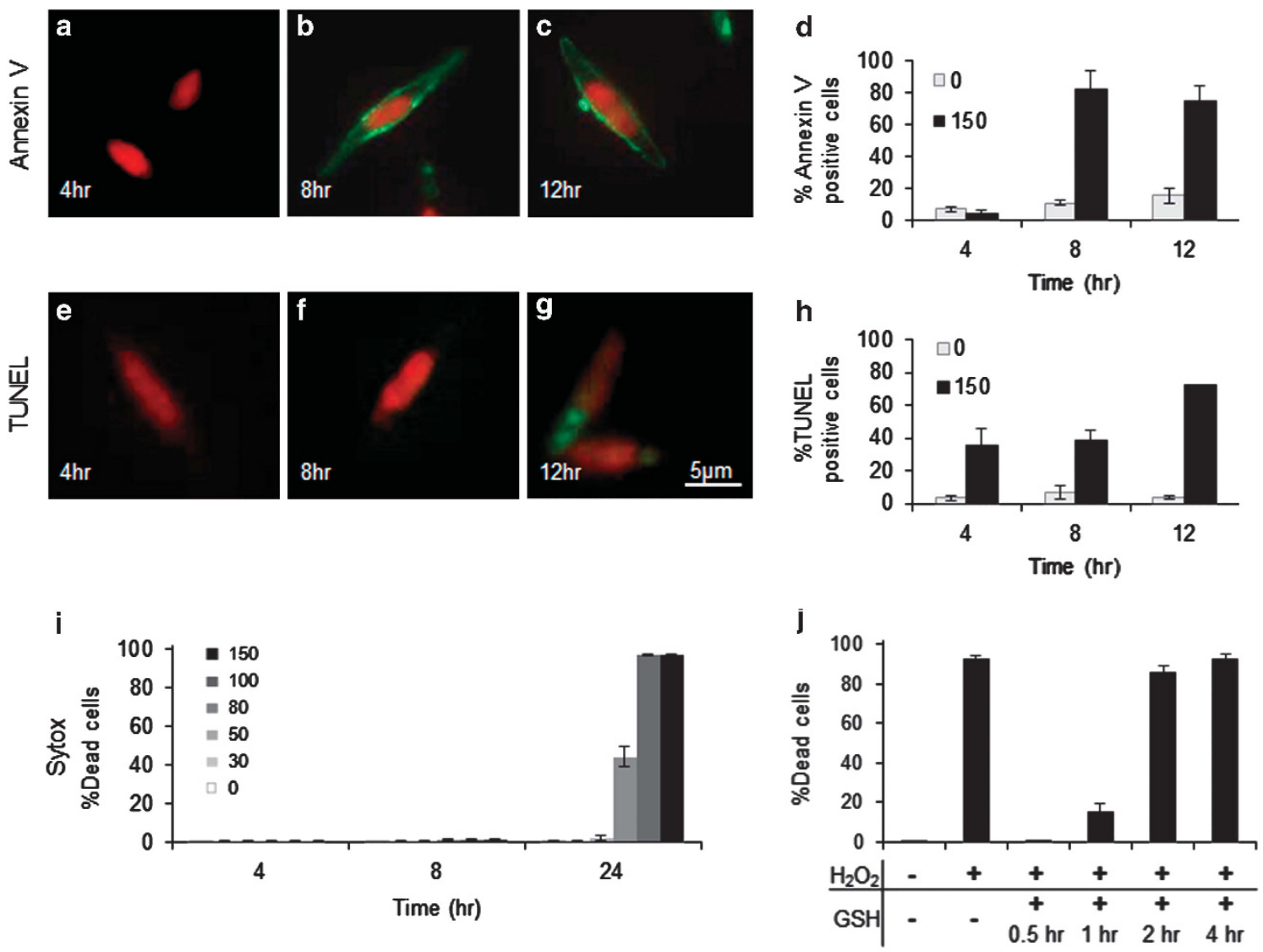

Figure 3 PCD in response to oxidative stress. (a-d) Phosphatidylserine externalization assessed by Annexin V 4, 8 and $12 \mathrm{~h}$ after application of $150 \mu \mathrm{M} \mathrm{H}_{2} \mathrm{O}_{2}$, viewed under the fluorescence microscope (a-c), and by flow cytometry in the green channel (d). (e-h) DNA fragmentations assessed by TUNEL assay 4,8 and $12 \mathrm{~h}$ after application of $150 \mu \mathrm{M} \mathrm{H}_{2} \mathrm{O}_{2}$, viewed under the fluorescence microscope (e-g) and by flow cytometry in the green channel (h). Green fluorescence represented in green, chlorophyll autofluorescence in red. Scale bar: $5 \mu \mathrm{m}$. (i) Cell death assessed as the fraction of Sytox green-positive cells 4, 8 and $24 \mathrm{~h}$ after treatment with 0, 30, 50, 80, 100 and 150 $\mu \mathrm{M}$ $\mathrm{H}_{2} \mathrm{O}_{2}$. (j) Cell death as measured $24 \mathrm{~h}$ after application of $1 \mathrm{~mm}$ GSH $0.5,1,2$ or $4 \mathrm{~h}$ after treatment with $150 \mu \mathrm{M} \mathrm{H}_{2} \mathrm{O}_{2}$. Flow cytometry analysis is based on fluorescence measurements of at least 5000 cells per sample and presented as mean \pm s.d., $n=3$. Representative data from at least three independent experiments, each containing three biological replicates, are shown.

$\mathrm{E}_{\mathrm{GSH}}$, we monitored subcellular $\mathrm{E}_{\mathrm{GSH}}$ oxidation in response to common stress conditions that diatom cells may be exposed to in the aquatic environment (Figure 4, Supplementary Table S1). We observed different responses to nitrogen and iron limitation, both are major nutrients, which limit diatom blooms in the ocean (Boyd et al., 2007; Moore et al., 2013). Although an increase of $64 \%$ in the chloroplast roGFP oxidation level was detected in response to nitrogen limitation, none of the examined organelles were significantly oxidized following nine days of iron limitation (Figure 4, Supplementary Table S1 and see also Rosenwasser et al., 2014). Exposure of the cells to high light $\left(700 \mu \mathrm{mol}\right.$ photons $\left.\mathrm{m}^{-2} \mathrm{~s}^{-1}\right)$ led to a significant oxidation of $11 \%$, exclusively in the chloroplast (Figure 4 and Supplementary Table S1).

We further exposed P. tricornutum to physiological concentrations of BrCN, which was recently shown to act as an allelochemical in benthic diatoms (Vanelslander et al., 2012). We detected roGFP oxidation in all the examined subcellular compartments in response to $0.5 \mu \mathrm{M}$, but not $0.1 \mu \mathrm{M}$
BrCN (Figure 4 and Supplementary Table S1). A cumulative, dose-dependent oxidation in the mitochondria was detected in response to the diatom-derived aldehyde 2E.4E/Z-decadienal (DD), an infochemical that is produced upon stress and is involved in chemical defense and intercellular signaling in diatoms (Ianora et al., 2004; Casotti et al., 2005; Vardi et al., 2006). Application of 50 and $100 \mu \mathrm{M}$ DD led to maximal mitochondrial $\mathrm{E}_{\mathrm{GSH}}$ oxidation of $40 \%$ and $90 \%$, respectively (Figure 4 and Supplementary Table S1). In contrast to the observed oxidation in the mitochondria, we detected only minor oxidation in the chloroplast, and slight dose-dependent oxidation in the nucleus in response to 5-100 $\mu \mathrm{M}$ DD. Maximal oxidation of $<9 \%$ in the chloroplast and $<13 \%$ in the nucleus were detected $3 \mathrm{~h}$ after treatment with $100 \mu \mathrm{M}$ DD (Figure 4 and Supplementary Table S1). Taken together, these data indicate specific compartmentalized redox modification patterns in response to environmental stress conditions (Figure 4 and Supplementary Table S1). These organelle-specific oxidation patterns may be involved in sensing the 


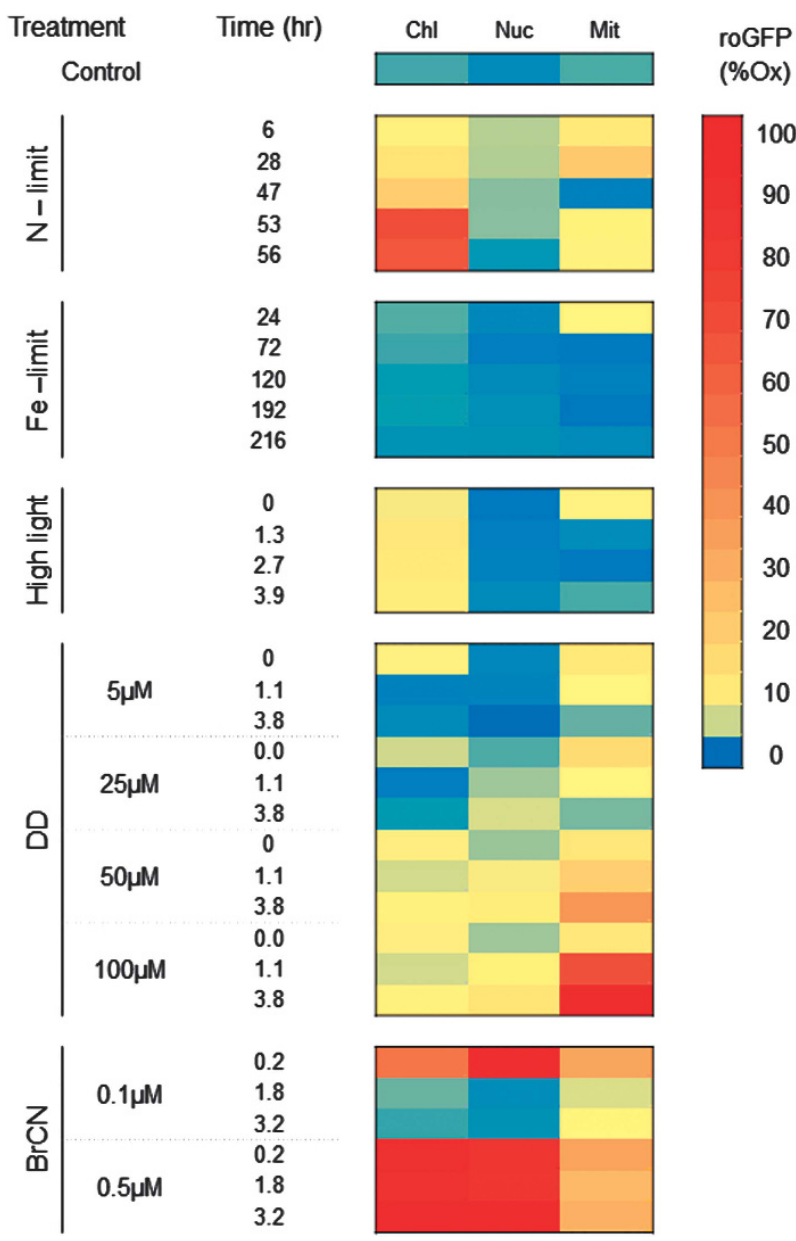

Figure 4 Organelle-specific oxidation patterns in response to environmental stress conditions. Heat map representation of roGFP degree of oxidation measured in the chloroplast (Chl), nucleus (Nuc) and mitochondria (Mit) in response to various environmental stress conditions. The stresses are: nitrogen and iron limitation ( $\mathrm{N}$ limit, $\mathrm{Fe}$ limit), exposure to high light $\left(700 \mu \mathrm{mol}\right.$ photons $\left.\mathrm{m}^{-2} \mathrm{~s}^{-1}\right)$ and to various concentration of the infochemicals DD and BrCN. roGFP degree of oxidation values were calculated based on averaged flow cytometry fluorescence measurements of at least 5000 cells per sample. Three biological replicated for each sample were measured. Representative data from at least three independent experiments, each containing three biological replicates, are shown. The actual average roGFP oxidation and SD values are provided as Supplementary Table S1.

cellular redox state under diverse environmental stress conditions and transmitting redox signals to respective targets.

Based on the observed interplay between early oxidation and subsequent cell death in response to $\mathrm{H}_{2} \mathrm{O}_{2}$ (Figures 2 and 3), we further linked early roGFP oxidation in specific organelles and cell death following diverse stress conditions. We plotted the maximal $E_{\mathrm{GSH}}$ in each organelle versus the fraction of sytox-positive cells $24 \mathrm{~h}$ after exposure to the examined stress conditions (Figures 5a-c). Using the K-means clustering approach, we revealed two distinct clusters, one for stress conditions that kept reduced $\mathrm{E}_{\mathrm{GSH}}$ and did not lead to activation of cell death after $24 \mathrm{~h}$ ('live' cluster), and another cluster composed of stresses that led to oxidized $\mathrm{E}_{\mathrm{GSH}}$ pool and induction of cell death ('dead' cluster). To quantify how well $\mathrm{E}_{\mathrm{GSH}}$ serves as a predictor of cell fate, we analyzed the overlap between the histograms of the 'live' and 'dead' clusters as a function of $E_{\mathrm{GSH}}$ (the $\mathrm{X}$ axis) in the various organelles (Figures $5 \mathrm{~d}-\mathrm{f}$, see also detailed explanation in Supplementary materials and methods). The Minimal overlap between the two clusters $(8 \%)$ was detected in the mitochondrial $\mathrm{E}_{\mathrm{GSH}}$, which indicates a strong correlation between early oxidation of the mitochondrial $\mathrm{E}_{\mathrm{GSH}}$ and subsequent induction of cell death (Figure 5c). Intriguingly, we revealed a distinct lifedeath threshold of $23 \%$ oxidation, corresponding to $-335 \mathrm{mV}$ in mitochondrial $\mathrm{E}_{\mathrm{GSH}}$, based on 200 samples obtained from application of various stress conditions (Supplementary Figure S3, Figures 5c, f and i). These results show a pivotal role for mitochondrial $\mathrm{E}_{\mathrm{GSH}}$ in cell fate determination and suggest that cell death can be precisely predicted based on the lifedeath mitochondrial $\mathrm{E}_{\mathrm{GSH}}$ threshold. We could not detect such a direct coupling between cell death and $\mathrm{E}_{\mathrm{GSH}}$ oxidation in the chloroplast or nucleus (Figures $5 \mathrm{a}$ and $\mathrm{b}, \mathrm{d}$ and e, g and h). For example, nitrogen limitation led to high oxidation in the chloroplast without subsequent induction of cell death, while high DD concentrations led to induction of cell death with specific oxidation in the mitochondria, without oxidation of the chloroplast (Figure 4, and compare Figures $5 \mathrm{~g}$ to 5i). Similarly, we did not observe a correlation between the $\mathrm{E}_{\mathrm{GSH}}$ in the nucleus and induction of cell death, as the lethal $\mathrm{DD}$ doses did not oxidize the nuclear $\mathrm{E}_{\mathrm{GSH}}$, and some of the BrCN treatments (250 nM) oxidized the nuclear $E_{\mathrm{GSH}}$ without induction of cell death (Figure 4, Figure 5h).

We further examined the role of early mitochondrial $\mathrm{E}_{\mathrm{GSH}}$ in mediating DD-induced cell death. The DD concentrations that led to early pronounced mitochondrial oxidation also led to extensive induction of cell death $24 \mathrm{~h}$ later (Figures 6a and b). Application of $1 \mathrm{~mm}$ GSH $30 \mathrm{~min}$ after treatment with the lethal concentration of $50 \mu \mathrm{M}$ DD, suppressed both mitochondrial oxidation and induction of cell death (Figures $6 \mathrm{~b}$ and c). Interestingly, this observed recovery was effective only within the 30-min time frame and at concentrations lower than $100 \mu \mathrm{M}$, corroborating our finding of a distinct critical phase of redox perturbation that determines the induction of downstream cell fate biochemical cascades.

Living within the chemo-physical gradient of light, nutrients and infochemicals, diatoms critically require mechanisms to rapidly respond to these diverse signals. Recent reports demonstrated the diatoms capabilities to perceive light, nutrients and infochemicals that are derived from trophiclevel interactions (Falciatore et al., 2000; Vardi et al., 2006; Coesel et al., 2009; Huysman et al., 2013). Modulation in the cellular $\mathrm{E}_{\mathrm{GSH}}$ in response to 

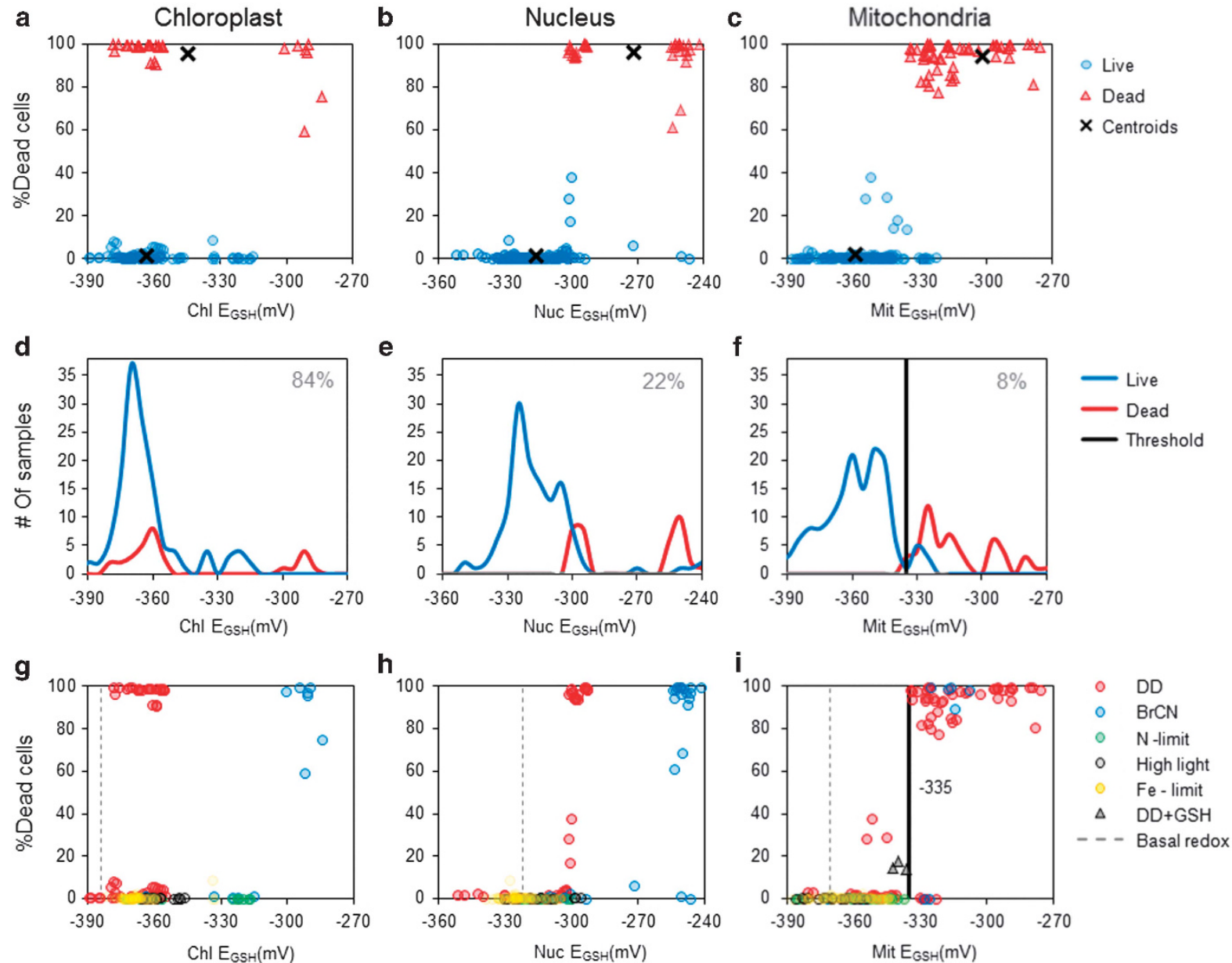

Figure 5 Mitochondrial $\mathrm{E}_{\mathrm{GSH}}$ predicts cell fate in response to environmental stresses. (a-c) Scatter plots of maximal $\mathrm{E}_{\mathrm{GSH}}$ oxidation (mV) in the chloroplast (a), the nucleus (b) and in the mitochondria (c) in response to a range of environmental stress conditions (as in Figure 4), and level of cell death $24 \mathrm{~h}$ after treatment. The clusters are marked as 'live' (blue circles) and 'dead' (red triangles), cluster centroids marked by X. (d-f) The normalized percent overlap of the two clusters along the $\mathrm{X}$ axis $\left(\mathrm{E}_{\mathrm{GSH}}\right)$ is presented as histograms of the 'live' and 'dead' clusters (blue and red lines, respectively) in the chloroplast (d), the nucleus (e) and in the mitochondria (f). (g-i) Same as in a-c, the different environmental stresses are marked in colors; dashed grey lines represent the basal $\mathrm{E}_{\mathrm{GSH}}$ under steady-state conditions. The black line represents the calculated mitochondrial redox threshold that can predict induction of cell death $24 \mathrm{~h}$ after maximal oxidation.

environmental stress conditions has been suggested as a key signaling cascade enabling the transduction of information regarding the cellular redox homeostasis into redox-sensitive proteins in order to activate or adjust specific biological functions (Meyer, 2008; Foyer and Noctor, 2011). Recent studies on several organisms including diatoms, used proteomic approaches to map the cellular redox-regulated protein network, which may represent the capability of the cells to transmit specific redox signals into biological pathways (Leichert et al., 2008; Brandes et al., 2011; Kumsta et al., 2011; Rosenwasser et al., 2014). In this study, we used the redox-sensitive GFP sensor targeted to specific organelles in order to map the in vivo spatial and temporal redox patterns in the model diatom $P$. tricornutum under steady state and in response to environmental stresses. We provide compelling evidence that diatoms respond differentially to diverse stresses such as nutrient limitation, oxidative stress and chemical signals derived from biotic interactions, by organelle-specific oxidation patterns (Figure 5). Modulations in the redox homeostasis of specific cellular compartments following stress, can lead to post-translation modifications in the redox state of redox-sensitive proteins in this microenvironment and by that transmit a specific redox signal into relevant biological pathways. For example, specific oxidation of the chloroplast $\mathrm{E}_{\mathrm{GSH}}$ under high light and nitrogen starvation can reflect oxidative stress conditions that affect the redox state of chloroplast targeted redox-sensitive proteins to adjust their biological function in order to maintain the cellular homeostasis under these environmental constraints (Dietz and Pfannschmidt, 2011; Dangoor et al., 2012).

By examining the interplay between organellespecific oxidation patterns and subsequent induction of cell death, we found a strong correlation between mitochondrial $\mathrm{E}_{\mathrm{GSH}}$ oxidation and cell 

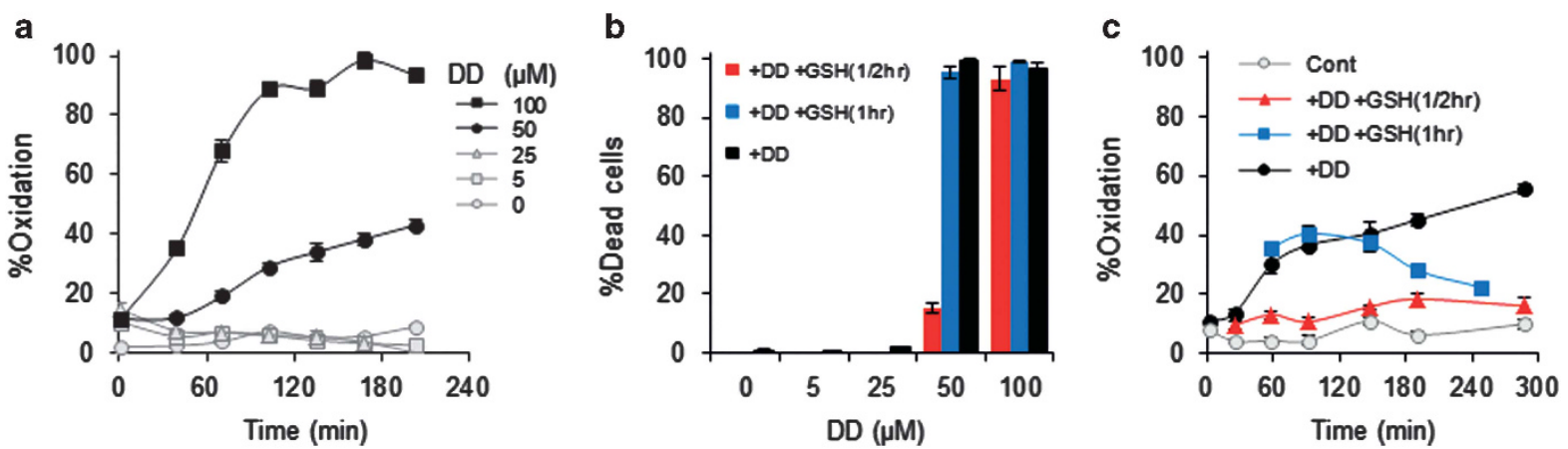

Figure 6 A reversible early mitochondrial $\mathrm{E}_{\mathrm{GSH}}$ oxidation mediates cell death in response to diatom-derived DD. (a) roGFP degree of oxidation in the mitochondria in response to $0,5,25,50$ and $100 \mu \mathrm{M}$ DD. (b) Induction of cell death $24 \mathrm{~h}$ after DD treatment (black bars), and upon additional treatment with $1 \mathrm{~mm}$ GSH after 30 or 60 min after treatment with DD (red and blue bars, respectively). (c) roGFP degree of oxidation in the mitochondria in response to $50 \mu \mathrm{M}$ DD (black circles), with or without additional application of 1 mM GSH after $30 \mathrm{~min}$ (red triangles) or $60 \mathrm{~min}$ (blue squares). Sytox staining for cell death assessment and roGFP degree of oxidation values were calculated based on averaged flow cytometry fluorescence measurements of at least 5000 cells per sample and presented as mean \pm s.d., $n=3$. Representative data from at least three independent experiments, each containing three biological replicates, are shown.

death and defined a distinct life-death threshold of mitochondrial $\mathrm{E}_{\mathrm{GSH}} \quad(-335 \mathrm{mV}, 23 \%$ oxidation $)$ (Figures $5 \mathrm{f}$ and i, Supplementary Figure S3). We further suggested a role for the oxidation state of mitochondrial $\mathrm{E}_{\mathrm{GSH}}$ in perception of DD-induced cell death. Mitochondria are recognized as having a central role in activation of ROS-dependent PCD in animals (Tait and Green, 2010; Rodriguez-Rocha et al., 2013) and oxidation of mitochondrial thioredoxin systems has been suggested as a common event in the initiation of PCD in animals and yeast (Zhang et al., 2004; Yin et al., 2012; Greetham et al., 2013). Nevertheless, very little is known about the molecular components involved in ROS perception and modulation and in execution of PCD in photosynthetic microorganisms. The absence of classical animal apoptotic components such as the Bcl-2 protein family and caspases suggests that although the mitochondria is a central mediator of PCD (Lam et al., 2001; Foyer and Noctor, 2003; Gadjev et al., 2008), the molecular pathways have diverged in animals and photosynthetic organisms. Moreover, even in animals, where the involvement of protein thiol status in mitochondrial permeability transition has been demonstrated (Kowaltowski et al., 2001), the proteins that mediate PCD by thiol oxidation are yet to be determined.

Taking into account the wide distribution of GSH as an antioxidant, possibly derived from a bacterial origin (Masip et al., 2006), it is possible that perturbation in $\mathrm{E}_{\mathrm{GSH}}$ is a conserved PCD signaling component (Koonin and Aravind, 2002; Bidle and Falkowski, 2004). Indeed, meta-analysis of plant seeds suggested $E_{G S H}$ as a universal marker for viability and cell death, whereby a shift of $\mathrm{E}_{\mathrm{GSH}}$ toward oxidation is correlated to the loss of seed viability (Kranner et al., 2006). Recently, the roGFP probe was used to identify a small fraction of susceptible neurons in a mouse model of Alzheimer's disease, in which cell death was preceded by oxidation of $\mathrm{E}_{\mathrm{GSH}}$ (Xie et al., 2013). However, in these studies, the specific subcellular localization of the $\mathrm{E}_{\mathrm{GSH}}$ oxidation was not examined. Interestingly, a recently published study in mammalian cell culture found a relationship between mitochondrial roGFP oxidation in response to parkinsonian neurotoxins and later cell death (Rodriguez-Rocha et al., 2013). Taken together, these observations suggest that GSH has a central and evolutionary conserved role in cell death signaling. Although the precise threshold value described here for cell death activation can be alerted in diatom cells that are acclimated to diverse physiological conditions (for example, highlight, temperature and low DD), it is possible that a distinct shift of the mitochondrial $\mathrm{E}_{\mathrm{GSH}}(30 \mathrm{mV})$ from its steady-state condition can predict the induction of cell death. It is possible that the mechanisms underlying the induction of PCD as a result of mitochondrial $\mathrm{E}_{\mathrm{GSH}}$ oxidation are common in a wide range of organisms, the identification of a distinct $\mathrm{E}_{\mathrm{GSH}}$ threshold can help to identity unknown components of the mitochondrial machinery that perceive the redox signal and initiate the cell death biochemical cascade.

\section{Conflict of Interest}

The authors declare no conflict of interest.

\section{Acknowledgements}

We thank Robert Fluhr for critical comments on the manuscript. We thank James Remington from University of Oregon for providing the roGFP gene. This research was supported by the European Research Council (ERC) StG (INFOTROPHIC grant \#280991) and the generous support of Edith and Nathan Goldenberg Career Development Chair to AV. 


\section{References}

Anning T, Nimer N, Merrett M, Brownlee C. (1996). Costs and benefits of calcification in coccolithophorids. J Mar Syst 9: 45-56.

Arfken GB, Weber H-J. (2000). Mathematical Methods for Physicists, 5th edn. Academic Press: Boston, MA, USA, pp 14-15.

Berges JA, Falkowski PG, York N. (1998). Physiological stress and cell death in marine phytoplankton: response to nitrogen or light limitation induction of proteases in response to nitrogen or light limitation. Limnol Oceanogr 43: 129-135.

Bidle KD, Falkowski PG. (2004). Cell death in planktonic, photosynthetic microorganisms. Nat Rev Microbiol 2: 643-655.

Boyd PW, Jickells T, Law CS, Blain S, Boyle Ea, Buesseler KO et al. (2007). Mesoscale iron enrichment experiments 1993-2005: synthesis and future directions. Science 315: 612-617.

Brandes N, Reichmann D, Tienson H, Leichert LI, Jakob U. (2011). Using quantitative redox proteomics to dissect the yeast redoxome. J Biol Chem 286: 41893-41903.

Casotti R, Mazza S, Brunet C, Vantrepotte V, Ianora A, Miralto A. (2005). Growth inhibition and toxicity of the diatom aldehyde 2-trans, 4-trans -decadienal on Thalassiosira Weissflogii (Bacillariophyceae). J Phycol 41: 7-20.

Coesel S, Mangogna M, Ishikawa T, Heijde M, Rogato A, Finazzi G et al. (2009). Diatom PtCPF1 is a new cryptochrome/photolyase family member with DNA repair and transcription regulation activity. EMBO Rep 10: $655-661$.

D’Autréaux B, Toledano MB. (2007). ROS as signalling molecules: mechanisms that generate specificity in ROS homeostasis. Nat Rev Mol Cell Biol 8: 813-824.

Dangoor I, Peled-Zehavi H, Wittenberg G, Danon A. (2012). A chloroplast light-regulated oxidative sensor for moderate light intensity in Arabidopsis. Plant Cell 24: 1894-1906.

Danon A. (2002). Redox reactions of regulatory proteins: do kinetics promote specificity? Trends Biochem. Science 27: 197-203.

Dardalhon M, Kumar C, Iraqui I, Vernis L, Kienda G, Banach-Latapy A et al. (2012). Redox-sensitive YFP sensors monitor dynamic nuclear and cytosolic glutathione redox changes. Free Radic Biol Med 52: 2254-2265.

Dietz K-J. (2008). Redox signal integration: from stimulus to networks and genes. Physiol Plant 133: 459-468.

Dietz K-J, Pfannschmidt T. (2011). Novel regulators in photosynthetic redox control of plant metabolism and gene expression. Plant Physiol 155: 1477-1485.

Dooley CT, Dore TM, Hanson GT, Jackson WC, Remington SJ, Tsien RY. (2004). Imaging dynamic redox changes in mammalian cells with green fluorescent protein indicators. $J$ Biol Chem 279: 22284-22293.

Falciatore A, D’Alcala MR, Croot P, Bowler C. (2000). Perception of environmental signals by a marine diatom. Science 288: 2363-2366.

Field C, Behrenfeld M, Randerson J, Falkowski P. (1998). Primary production of the biosphere: integrating terrestrial and oceanic components. Science 281: $237-240$.

Foyer C, Noctor G. (2011). Ascorbate and glutathione: the heart of the redox hub. Plant Physiol 155: 2-18.
Foyer C, Noctor G. (2003). Redox sensing and signalling associated with reactive oxygen in chloroplasts, peroxisomes and mitochondria. Physiol Plant 119: 355-364.

Franklin DJ, Brussaard CPD, Berges Ja. (2006). What is the role and nature of programmed cell death in phytoplankton ecology? Eur J Phycol 41: 1-14.

Gadjev I, Stone JM, Gechev TS. (2008). Programmed cell death in plants: new insights into redox regulation and the role of hydrogen peroxide. In International Review of Cell and Molecular Biology, Vol. 270. Elsevier Inc.: San Diego, CA, USA; Waltham, MA, USA; Oxford, UK; London, UK; Amsterdam, The Netherlands, pp 87-144.

Gadjev I, Vanderauwera S, Gechev sanko S, Laloi C, Minkov IN, Shulaev V et al. (2006). Transcriptomic footprints disclose specificity of reactive oxygen species signaling in Arabidopsis. Plant Physiol 141: $436-445$.

Greetham D, Kritsiligkou P, Watkins RH, Carter Z, Parkin J, Grant CM. (2013). Oxidation of the yeast mitochondrial thioredoxin promotes cell death. Antioxid Redox Signal 18: 376-385.

Guillard R, Ryther J. (1962). Studies of marine planktonic diatoms. I. Cyclotella nana Hustedt, and Detonula confervacea (cleve) Gran. Can J Microbiol 8: 229-239.

Gutscher M, Pauleau A, Marty L, Brach T, Wabnitz GH, Samstag Y et al. (2008). Real-time imaging of the intracellular glutathione redox potential. Nat Methods 5: 553-559.

Halliwell B. (2006). Reactive species and antioxidants. Redox biology is a fundamental theme of aerobic life. Plant Physiol 141: 312-322.

Hanson GT, Aggeler R, Oglesbee D, Cannon M, Capaldi Ra, Tsien RY et al. (2004). Investigating mitochondrial redox potential with redox-sensitive green fluorescent protein indicators. J Biol Chem 279: 13044-13053.

$\mathrm{Hu}$ J, Dong L, Outten CE. (2008). The redox environment in the mitochondrial intermembrane space is maintained separately from the cytosol and matrix. J Biol Chem 283: 29126-29134.

Huysman MJJ, Fortunato AE, Matthijs M, Costa BS, Vanderhaeghen R, Van den Daele H et al. (2013). AUREOCHROME1a-mediated induction of the diatom-specific cyclin dsCYC2 controls the onset of cell division in diatoms (Phaeodactylum tricornutum). Plant Cell 25: 215-228.

Ianora A, Miralto A, Poulet SA, Carotenuto Y, Buttino I, Romano G et al. (2004). Aldehyde suppression of copepod recruitment in blooms of a ubiquitous planktonic diatom. Nature 429: 403-407.

Jones DP, Go Y-M. (2010). Redox compartmentalization and cellular stress. Diabetes Obes Metab 12: 116-125.

Koonin EV, Aravind L. (2002). Origin and evolution of eukaryotic apoptosis: the bacterial connection. Cell Death Differ 9: 394-404.

Kowaltowski AJ, Castilho RF, Vercesi AE. (2001). Mitochondrial permeability transition and oxidative stress. FEBS Lett 495: 12-15.

Kranner I, Birtić S, Anderson KM, Pritchard HW. (2006). Glutathione half-cell reduction potential: a universal stress marker and modulator of programmed cell death? Free Radic Biol Med 40: 2155-2165.

Kumar C, Igbaria A, D’Autreaux B, Planson A-G, Junot C, Godat E et al. (2011). Glutathione revisited: a vital function in iron metabolism and ancillary role in thiol-redox control. EMBO J 30: 2044-2056. 
Kumsta C, Thamsen M, Jakob U. (2011). Effects of oxidative stress on behavior, physiology, and the redox thiol proteome of Caenorhabditis elegans. Antioxid Redox Signal 14: 1023-1037.

Lam E, Kato N, Lawton M. (2001). Programmed cell death, mitochondria and the plant hypersensitive response. Nature 411: 848-853.

Leichert LI, Gehrke F, Gudiseva HV, Blackwell T, Ilbert M, Walker AK et al. (2008). Quantifying changes in the thiol redox proteome upon oxidative stress in vivo. Proc Natl Acad Sci USA 105: 8197-8202.

Masip L, Veeravalli K, Georgiou G. (2006). The many faces of glutathione in bacteria. Antioxid Redox Signal 8: $753-762$

Meyer AJ. (2008). The integration of glutathione homeostasis and redox signaling. J Plant Physiol 165: 1390-1403.

Meyer AJ, Brach T, Marty L, Kreye S, Rouhier N, Jacquot J-P et al. (2007). Redox-sensitive GFP in Arabidopsis thaliana is a quantitative biosensor for the redox potential of the cellular glutathione redox buffer. Plant J 52: 973-986.

Meyer AJ, Dick TP. (2010). Fluorescent protein-based redox probes. Antioxid Redox Signal 13: 621-650.

Mittler R. (2002). Oxidative stress, antioxidants and stress tolerance. Trends Plant Sci 7: 405-410.

Mittler R, Vanderauwera S, Gollery M, Van Breusegem F. (2004). Reactive oxygen gene network of plants. Trends Plant Sci 9: 490-498.

Møller IM, Sweetlove LJ. (2010). ROS signalling specificity is required. Trends Plant Sci 15: 370-374.

Moore CM, Mills MM, Arrigo KR, Berman-Frank I, Bopp L, Boyd PW et al. (2013). Processes and patterns of oceanic nutrient limitation. Nat Geosci 6: 701-710.

Nelson DMD, Trrguer P, Brzezinski MA, Leynaert A, Queguiner B, Tréguer P. (1995). Production and dissolution of biogenic silica in the ocean: revised global estimates, comparison with regional data and relationship to biogenic sedimentation. Glob Biogeochem Cycle 9: 359-372.

Orellana MV, Pang WL, Durand PM, Whitehead K, Baliga NS. (2013). A role for programmed cell death in the microbial loop. PLoS One 8: e62595.

Pohnert G, Steinke M, Tollrian R. (2007). Chemical cues, defence metabolites and the shaping of pelagic interspecific interactions. Trends Ecol Evol 22: 198-204.

Rijstenbil J, Wijnholds J. (1996). HPLC analysis of nonprotein thiols in planktonic diatoms: pool size, redox state and response to copper and cadmium exposure. Mar Biol 127: 45-54.

Rodriguez-Rocha H, Garcia-Garcia A, Pickett C, Li S, Jones J, Chen $\mathrm{H}$ et al. (2013). Compartmentalized oxidative stress in dopaminergic cell death induced by pesticides and complex I inhibitors: distinct roles of superoxide anion and superoxide dismutases. Free Radic Biol Med 61C: 370-383.

Rosenwasser S, Fluhr R, Joshi JR, Leviatan N, Sela N, Hetzroni A et al. (2013). ROSMETER: a bioinformatic tool for the identification of transcriptomic imprints related to reactive oxygen species type and origin provides new insights into stress responses. Plant Physiol 163: 1071-1083.
Rosenwasser S, Graff van Creveld S, Schatz D, Malitsky S, Tzfadia O, Aharoni A et al. (2014). Mapping the diatom redox-sensitive proteome provides insights into response to nitrogen stress in the marine environment. Proc Natl Acad Sci USA 111: 2740-2745.

Rosenwasser S, Rot I, Meyer AJ, Feldman L, Jiang K, Friedman H. (2010). A fluorometer-based method for monitoring oxidation of redox-sensitive GFP (roGFP) during development and extended dark stress. Physiol Plant 138: 493-502.

Schwarzländer M, Fricker MD, Müller C, Marty L, Brach T, Novak J et al. (2008). Confocal imaging of glutathione redox potential in living plant cells. J Microsc 231: 299-316.

Tait SWG, Green DR. (2010). Mitochondria and cell death: outer membrane permeabilization and beyond. Nat Rev Mol Cell Biol 11: 621-632.

Thamatrakoln K, Korenovska O, Niheu AK, Bidle KD. (2012). Whole-genome expression analysis reveals a role for death-related genes in stress acclimation of the diatom Thalassiosira pseudonana. Environ Microbiol 14: $67-81$.

Vanelslander B, Paul C, Grueneberg J, Prince EK, Gillard J, Sabbe K et al. (2012). Daily bursts of biogenic cyanogen bromide (BrCN) control biofilm formation around a marine benthic diatom. Proc Natl Acad Sci USA 109: 2412-2417.

Vardi A, Bidle KD, Kwityn C, Hirsh DJ, Thompson SM, Callow Ja et al. (2008). A diatom gene regulating nitricoxide signaling and susceptibility to diatom-derived aldehydes. Curr Biol 18: 895-899.

Vardi A, Formiggini F, Casotti R, De Martino A, Ribalet F, Miralto A et al. (2006). A stress surveillance system based on calcium and nitric oxide in marine diatoms. PLoS Biol 4: e60.

Xie H, Hou S, Jiang J, Sekutowicz M, Kelly J, Bacskai BJ. (2013). Rapid cell death is preceded by amyloid plaque-mediated oxidative stress. Proc Natl Acad Sci USA 110: 7904-7909.

Yin F, Sancheti H, Cadenas E. (2012). Mitochondrial thiols in the regulation of cell death pathways. Antioxid Redox Signal 17: 1714-1727.

Zhang R, Al-Lamki R, Bai L, Streb JW, Miano JM, Bradley J et al. (2004). Thioredoxin-2 inhibits mitochondrialocated ASK1-mediated apoptosis in a JNK-independent manner. Circ Res 94: 1483-1491.

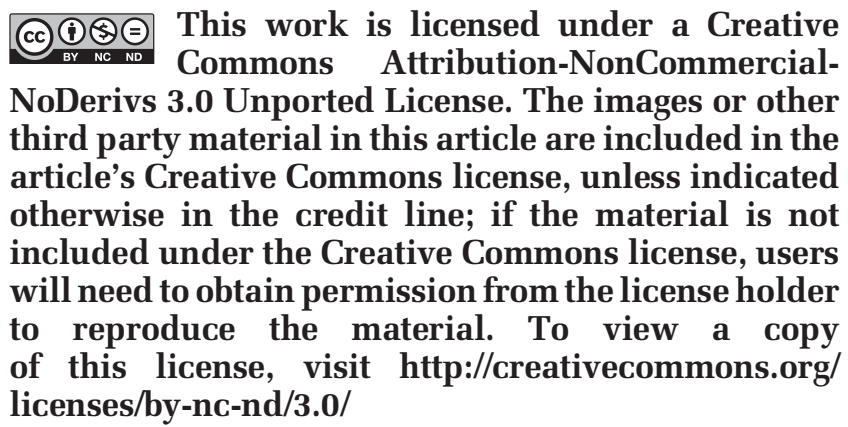

Supplementary Information accompanies this paper on The ISME Journal website (http://www.nature.com/ismej) 